\title{
An Automated Cell Line Authentication Method for AstraZeneca Global Cell Bank using Deep Neural Networks on Brightfield Images
}

\section{Lei Tong}

School of Computing and Mathematical Sciences, University of Leicester

\section{Adam Corrigan}

Data Sciences \& Quantitative Biology, Discovery Sciences, AstraZeneca R\&D, Cambridge

Navin Rathna Kumar

UK Cell Culture and Banking, Discovery Sciences, AstraZeneca R\&D, Alderley Park

\section{Kerry Hallbrook}

UK Cell Culture and Banking, Discovery Sciences, AstraZeneca R\&D, Alderley Park

\section{Jonathon Orme}

UK Cell Culture and Banking, Discovery Sciences, AstraZeneca R\&D, Alderley Park

\section{Yinhai Wang ( $\triangle$ Yinhai.Wang@astrazeneca.com)}

Data Sciences \& Quantitative Biology, Discovery Sciences, AstraZeneca R\&D, Cambridge

Huiyu Zhou

School of Computing and Mathematical Sciences, University of Leicester

\section{Research Article}

Keywords: cell line authentication, cell bank, deep neural network, machine learning, artificial intelligence

Posted Date: October 12th, 2021

DOl: https://doi.org/10.21203/rs.3.rs-956546/v1

License: (c) (i) This work is licensed under a Creative Commons Attribution 4.0 International License.

Read Full License 
Article

An Automated Cell Line Authentica-

tion Method for AstraZeneca Global

Cell Bank using Deep Neural Networks

on Brightfield Images

Lei Tong $1,2, *$ Adam Corrigan ${ }^{2, *}$ • Navin Rathna Kumar ${ }^{3} \cdot$ Kerry

Hallbrook $^{3} \cdot$ Jonathon Orme $^{3} \cdot$ Yinhai Wang $^{2} \cdot$ Huiyu Zhou $^{1}$

1 School of Computing and Mathematical Sciences, University of Leices-

ter, United Kingdom

2 Data Sciences \& Quantitative Biology, Discovery Sciences, AstraZeneca

R\&D, Cambridge, UK

3 UK Cell Culture and Banking, Discovery Sciences, AstraZeneca R\&D,

Alderley Park, UK

These authors contributed equally to this work

Correspondence should be addressed to Yinhai Wang

Key words: cell line authentication, cell bank, deep neural network, machine learning, artificial intelligence 


\section{Abstract:}

Cell line authentication is important in the biomedical field to ensure that researchers are not working with misidentified cells. Short tandem repeat is the gold standard method, but has its own limitations, including being expensive and time-consuming. Deep neural networks achieve great success in the analysis of cellular images in a costeffective way. However, because of the lack of centralized available datasets, whether or not cell line authentication can be replaced or supported by cell image classification is still a question. Moreover, the relationship between the incubation times and cellular images has not been explored in previous studies. In this study, we automated the process of the cell line authentication by using deep learning analysis of brightfield cell line images. We proposed a novel multi-task framework to identify cell lines from cell images and predict the duration of how long cell lines have been incubated simultaneously. Using thirty cell lines' data from the AstraZeneca Cell Bank, we demonstrated that our proposed method can accurately identify cell lines from brightfield images with a $99.8 \%$ accuracy and predicts the incubation durations for cell images with the coefficient of determination score of 0.927 . Considering that new cell lines are continually added to the AstraZeneca Cell Bank, we integrated the transfer learning technique with the proposed system to deal with data from new cell lines not included in the pre-trained model. Our method achieved excellent performance with a sensitivity of $97.7 \%$ and specificity of $95.8 \%$ in the detection of 14 new cell lines. These results demonstrated that our proposed framework can effectively identify cell lines using brightfield images. 


\section{Introduction}

Over the last 50-60 years, cell lines have become a staple of biological research, resulting in rapid developments in the fields of cell and molecular biology, however, this increased use of cell lines has brought to light the issue of cell line authentication [1]]. Many isolated cell lines were subsequently found to be contaminated by faster growing cell cultures, such as HeLa cells, due to poor cell culture practice leading to the misidentification of cell lines [1]. The use of a misidentified cell line can lead to false conclusions and irreproducible experiments, consequently leading to a waste of time, money and resource $[\underline{2}, \underline{3}]$. It is estimated that industry wide $10-20 \%$ of preclinical effort was wasted due to misidentified cell lines, estimated to cost the industry 28 billion USD per year [ $\underline{4}]$.

Though there are many methods for cell line authentication, short tandem repeat (STR) profiling, also sometimes referred to as DNA fingerprinting, has been the most widely used and is recommended as the standard by the American Type Culture Collection (ATCC) Standards Development Organization Workgroup ASN-0002 []ㅡ. In spite of the prevalence of STR profiling, there are limitations. Microsatellite instability and loss of heterozygosity, especially in cancer cell lines, can make validation and authentication challenging using STR profiling []]. In one study involving hematopoietic cancer cell 
lines it was found that the effect of long term culture, subcloning, and selection led to genetic drift, thereby significantly altering the DNA fingerprinting and over time some cell lines may also go through genetic and transcriptional evolution (GTE) [표 $\underline{8}]$. All of these variances can make it harder to discriminate between cell lines using STR profiling []]. In addition, due to time and cost restrictions, standard practice is to test the cells once they have been fully expanded and frozen, however this process results in wasted time and effort if the sample then fails STR profiling. It was therefore desirable to develop a new authentication approach to complement STR testing. Ideally, the new method should be easy to use, quick, and cost effective, and would enable early identification of the cell line that could be built into standard laboratory practice as well as overcoming the limitations of STR profiling $[\underline{9}, \underline{10}]$.

With the advancements of deep learning (DL) approaches, automated cell image classification is a possible solution to allow fast analysis at low cost, as well as potentially identifying changes in cell morphology that could be indicative of undesirable qualities such as genetic drift or cellular senescence. Oei et al. collected 522 fluorescence cell images from 3 cell lines (e.g. MCF10A, MCF-7, MDA-MB-231) using confocal immunofluorescence microscopy [11]. By using the features of actin cytoskeleton structures, they proposed a convolutional neural network (CNN) based on VGG-16 to classify 
the microscopy images into three categories and reported the performance of CNN outperforms their biological experts in the classification task. Akogo et al. used MobileNet to perform image classification in their 5 cell line dataset (MDA-MB-468, MCF7, 10A, 12A and HC11) with 96.67\% accuracy [12]. Most recently, Mzurikwao et al. trained two CNNs to classify 4 cancer cell lines (COLO 704, UKF-NB-3, EFO-27 and EFO-21) and their isogenic cell lines using brightfield images [10]. Compared with STR profiling, deep learning methods improve the cost and efficiency as these methods only need cellular images to train CNNs and the trained models are used to directly predict the identities for held-out test data. However, because of the lack of centralized available datasets and the intrinsic difficulties in analyzing multi-batch cellular images from different cell lines over time, the previous studies only conducted their experiments on small-scale cell line datasets of no more than ten cell lines. Whether or not CNN can identify tens even hundreds of cell lines is an open question. The relationship between the incubation times and cellular images has not been analyzed in previous studies. This could be tremendously useful for flagging growth rate defects or analyzing the differentiation processes of stem cells in the future. In addition, new cell lines are continually added to the AstraZeneca Cell Bank therefore, how to deal with data from new cell lines not included in the model requires consideration. 
Therefore, in this study, we aim to answer these questions by developing an automated cell line authentication method by using deep learningbased analysis of routine brightfield cell line images. Our main contributions reported in this paper are:

(1) Dataset: We have established two cellular datasets. i) The first dataset consists of 47671 brightfield images of 30 cell lines, which is $23 \mathrm{~GB}$ of data. The dataset was curated from a set of experiments where 30 cell lines were cultured and each cell culture flask was imaged at 2-3 hour intervals using the IncuCyte. To our knowledge, it is one of the largest such collections of data in the literature for cell line authentication. ii) The second dataset includes 860 cell images from 14 new cell lines not included in the 30 above. The 14 cell lines were incubated by using the same conditions as the previous 30 cell lines and we used this dataset to validate whether or not our proposed method can identify new cell lines without training model from scratch.

(2) Cell image recognition framework: We proposed a novel multi-task cell image recognition framework to i) identify and authenticate cell lines and ii) predict the duration of how long cell lines have been incubated simultaneously. The cell line classification network (CLCNet) learns image-level features from the cell images and outputs the predicted probabilities of the cell line labels for each input image. The extracted 
convolutional features of CLCNet are integrated with the cell line regression network (CLRNet) to predict the incubated time points for bright-field images.

(3) Identify new cell lines: We integrated a transfer learning approach with the proposed framework to identify images from new cell lines which are not included in the pre-trained model (currently we have 30 cell lines). This is an important aspect, as we are unlikely to be able to acquire very large numbers of images for every cell line that we wish to authenticate.

(4) Validation: We conducted comprehensive validation experiments to justify the significance of our proposed framework on the established datasets. The prediction performance of CLCNet reached the accuracy of $99.8 \%$ and the $\mathrm{fl}$-score of $99.7 \%$ in the classification of the 30 cell lines. CLRNet achieved the coefficient of determination score (R2score) of 0.927 in the prediction of the incubated times for cell images. In the detection of 14 new cell lines, our proposed method obtained the sensitivity of $97.7 \%$ and the specificity of $95.8 \%$. These results demonstrated that our proposed framework can effectively authenticate the identities of cell lines from brightfield images. 


\section{Materials and Methods}

\section{Cell Culture and Image Acquisition}

We have established two cell line datasets. (1) The first dataset consists of 30 different cell lines, as listed in Table. S1. The base medium is listed in the table, all medium was supplemented with $10 \%$ Foetal Bovine Serum (Sigma) and 1x GlutaMAX (Gibco) unless otherwise stated. The cells were thawed and seeded into a $25 \mathrm{~cm}^{2}$ flask (Corning) at a density of $0.5-2 \times 10^{6}$ cells per flask. The flasks were then added to the Incucyte S3 (Essen Bioscience, Sartorius) and phase contrast images collected from 21 different locations across the flask every 2-3 hours over a time period between 3-7 days.

(2) The second dataset includes images from 14 cell lines, as listed in Table. S2, these images were not used in the training of the CNN network. In this dataset, each cell line includes one location only resulting in fewer images. All images were exported for analysis as JPEGs or TIFF format using the Incucyte S3 software with $1408 \times 1040$ resolution. Example images of three cell lines (e.g. A431, A549, T47D) with different incubated durations are shown in Fig. 1.

\section{Multi-task Framework Overview}

Our proposed framework is shown in Fig. 2. In the data preparation stage, cell images are collected using the IncuCyte for all 30 cell lines. Each cell image has two separate labels, cell line name and incubation time. Then, the deep learning network CLCNet learns the image-level features from the input cell images with their cell line labels and outputs the predicted classes for test cell images. Once CLCNet is trained, the convolutional features of the training data are extracted to train CLRNet. CLRNet predicts the time of incubation after initial seeding for each image. 
Before the model training of CLCNet, we carried out the following pre-processing procedures: (1) Image scaling: The original image resolution for the established dataset is $1408 \times 1040$. In the training dataset, each cell image is cropped at a random region with the size $896 \times 869$ to improve the data diversity, which can influence the generalization and robustness of the training model in the downstream tasks. Images from the test set are cropped at a fixed center region with the same size $896 \times 869$ for later method comparison experiments. The cropped images are resized to $224 \times 224$ using the bilinear interpolation [13]. (2) Grayscale: The scaled cell images are gray but have three channels. The grayscale method is applied to convert the images into one channel. (3) Data Normalization: Data normalization aims to restrict the image pixels within a specific range and ensure each pixel has similar data distribution [10]. Each cell image is normalized by subtracting the image mean (i.e. pixel values) and dividing by image standard deviation, to improve the convergence speed while training the neural networks.

\section{Cell Line Classification Network}

We employed the Xception model [14] as the backbone of CLCNet for classification. The model structure used in our system is shown in Fig. S1. After pre-processing procedures, the input shapes of the cell images are $224 \times 224 \times 1$ (Height $\times$ Width $\times$ Channel). The input images will first pass through two convolution layers that each convolutional layer is followed by batch normalization [피 ] and a ReLU activation function. Five depthwise separable convolutional (DSC) blocks are stacked to further learn spatial and hierarchical features from the inputs. Each DSC block includes two or three DSC layers and one maxpooling layer. DSC layer consists of a depthwise convolution (i.e. a spatial convolution performed independently over every channel of input) and a pointwise convolution (i.e. a convolution with 
$1 \times 1$ kernel, projecting the channels computed by the depthwise convolution onto a new channel space) [16]. The mathematical formulation is defined as:

$$
\mathrm{O}_{\mathrm{k}, \mathrm{l}, \mathrm{E}}=\sum_{m}^{M} \widetilde{K}_{m, E} \cdot \sum_{i, j}^{I, J} K_{i, j, m} \odot F_{i+k, j+l, m}
$$

where $\mathrm{O}$ is the output feature map, $k \times l$ is the kernel size of the depthwise convolution and $\mathrm{E}$ is the channel of the output feature map. On the right hand side of Eq. (1), $\widetilde{K}$ and $K$ are the convolutional filters of the pointwise and depthwise convolution separately. $F$ is the input feature map and $\mathrm{I} \times \mathrm{J} \times \mathrm{M}$ is the shape of the input feature map. $\odot$ represents the operation of an element-wise product. The output of the DSC blocks is concatenated with the output of a shortcut convolution $(1 \times 1 \mathrm{Conv})$ through the residual connection. Only the fourth DSC block's feature map concatenates with the input feature map without the convolutional processing. The dimension of the output feature map from the final two DSC layers is $7 \times 7 \times 2048$ and the feature map is converted to 2048 dimensions by the adaptive average pooling layer. The final fully connected layer outputs the probability distribution for 30 cell lines. The classification loss $\mathcal{L}_{c l c}$ is computed using the cross entropy:

$$
\mathcal{L}_{c l c}=-\frac{1}{C} \sum_{c=1}^{c} \frac{1}{B} \sum_{X_{b} \in X} 1\left\{\Upsilon_{b}^{c}=c\right\} \log \left(\mathrm{P}\left(\Upsilon_{b}^{c}=c \mid X_{b} ; W\right)\right)
$$

where $C$ is the number of class and $B$ is the batch size. $X_{b}$ represents the $b$-th sample in the batch and $1\{\cdot\}$ denotes a characteristic function that 
$1\{\cdot\}=1$ if the condition is true and 0 otherwise. $\mathrm{P}\left(\Upsilon_{b}^{c}=c \mid X_{b} ; W\right)$ is the probability of the sample $X_{b}$ being correctly predicted as the class $\Upsilon_{b}^{c}$ using the network parameters $W$.

During the network training, $10 \%$ of data are split from the training set for validation. The performance of Xception model on the validation set is monitored for each 5 training epochs. Beyond epoch 50, if the validation loss has not decreased for 10 consecutive epochs, early stopping is triggered and the best model with the lowest validation loss is used for reporting the performance on the held-out test set.

\section{Cell Line Regression Network}

Network features, F, are extracted from the adaptive average pooling layer and its dimension is 2048 . We employed a multilayer perceptron (MLP) [17] as the backbone of CLRNet to refine features and reduce the dimension finally predict the incubation times for cell images. The network consists of three FC layers. The first two layers include 512 and 128 neurons separately and each layer is followed by a ReLU activation function. The third FC layer has 16 neurons and its output feature vectors are then transformed to a scalar which is the prediction result of the regression task. We add dropout units [1] with 0.2 rates after all FC layers to avoid overfitting. The regression loss $\mathcal{L}_{c l r}$ is computed using the mean squared error (MSE):

$$
\mathcal{L}_{c l r}=\frac{1}{B} \sum_{\mathrm{F}_{b} \in \mathrm{F}}\left(\mathrm{T}_{b}-\Gamma\left(\mathrm{F}_{b} ; \bar{W}\right)\right)^{2}
$$

where $T_{b}$ is the ground truth and $\Gamma\left(\mathrm{F}_{b} ; \bar{W}\right)$ is the predicted incubation times for cell images. Similar to the training strategy of CLCNet, we also randomly selected $10 \%$ of data for the validation of CLRNet. The validation 
MSE loss is monitored each epoch and the best model with the lowest validation loss is saved after 50 epochs.

\section{Transfer Learning for Identifying New Cell Lines}

To deal with data from new cell lines which are not included in the pretrained model, combining the previous data with the new obtained data and retraining CLCNet from scratch is a way to solve this issue. However, the time and computational resources required are prohibitive for this approach. Hence, we integrated the transfer learning technique with CLCNet to identify new cell lines. We first take the pre-trained CLCNet model which is trained on the 30 cell lines dataset and freeze all layers except the last FC layer. The FC layer is replaced by a new FC layer with 44 neurons (i.e. 30 original cell lines +14 new cell lines). The weights of the new FC layer are initialized. During the model training, only the weights of the FC layer will be updated with new data and the weights of other layers are fixed. The training set of the 30 cell lines is combined with the training set of the 14 cell lines. The underlying concept is that having been trained on 30 cell lines, the model will have learned a good representation of brightfield images for discriminating between different cell lines, and as such good classification performance can be obtained for new cell lines by only retraining the final classification layer. The combined test set is used to validate whether or not the updated model can keep good classification performance on the 30 cell lines and identify the new 14 cell lines.

\section{Results}

\section{Implementation Details}

The hyperparameters for CLCNet and CLRNet were set as follows: The SGD optimizer with the momentum $=0.9$ and weight_decay $=5 \mathrm{e}-4$ is adopted. The initial learning rate is 0.001 and it decays by factor $=0.1$ 
every 25 epochs. The batch size and maximum training epochs are 20 and 100 separately. To evaluate the model performance, 5 -fold crossvalidation [19] was applied. We implemented all models and benchmark experiments using Python 3.7 with Pytorch 1.9.0 [20] and Scikit-learn 0.24.0 [21] packages. We deployed all the experiments using a single 32GB Nvidia V100 GPU.

\section{Cell line Classification and Regression}

In order to evaluate our proposed system comprehensively, we replaced the backbone of CLCNet with three SOTA CNNs: MobileNet, VGG19, ResNet50. The results of the classification and regression on the 30 cell lines are shown in Table. 1. We evaluated the classification performance of CLCNet with four metrics, namely accuracy, precision, recall and f1-score:

$$
\begin{gathered}
\text { accuracy }=\frac{T P+T N}{T P+F N+T N+F P} \\
\text { precision }=\frac{T P}{T P+F P} \\
\text { recall }=\frac{T P}{T P+F N} \\
\mathrm{f} 1=\frac{2 \times \text { precision } \times \text { recall }}{\text { precision }+ \text { recall }}
\end{gathered}
$$

where $\mathrm{TP}$ is true positive, $\mathrm{TN}$ is true negative, $\mathrm{FN}$ is false negative and $\mathrm{FP}$ is false positive. The Xception model obtained the best classification performance and achieved the average accuracy of $99.8 \%$ and the f1-score of $99.7 \%$ across the 5-fold cross-validation. The classification features of the four backbones were extracted to train the CLRNet separately. The regression performance is evaluated by two metrics, MSE and R2-score:

$$
\operatorname{MSE}=\frac{1}{\mathrm{~N}} \sum_{\mathrm{n}=1}^{\mathrm{N}}\left(\mathrm{Y}_{\mathrm{n}}-\widehat{\mathrm{Y}}_{\mathrm{n}}\right)^{2}
$$




$$
\mathrm{R} 2=1-\frac{\sum_{\mathrm{n}=1}^{\mathrm{N}}\left(\mathrm{Y}_{\mathrm{n}}-\widehat{\mathrm{Y}}_{\mathrm{n}}\right)^{2}}{\sum_{\mathrm{n}=1}^{\mathrm{N}}\left(\mathrm{Y}_{\mathrm{n}}-\overline{\mathrm{Y}}\right)^{2}}
$$

where $\mathrm{N}$ is the number of test samples. $\mathrm{Y}_{\mathrm{n}}$ is the real value of the $\mathrm{n}$-th sample and $\widehat{Y}_{\mathrm{n}}$ is the predicted value. $\overline{\mathrm{Y}}$ denotes the average of all samples. The overall MSE and R2-score of Xception for the incubation duration prediction were 262.283 and 0.931 separately. We further demonstrated the classification results by using the confusion matrix in Fig. 3A, which shows that Xception made fewer prediction errors than ResNet50, VGG19, and MobileNet. The regression results of the test fold- 1 are visualized by using the scatter plots, Fig. 3B. Xception outperformed the other three methods in predicting the amount of the incubation times with $\mathrm{R} 2$-score $=0.939$. The complete regression results of the 5-fold cross-validation are shown in Fig. S2.

To further view how the deep network distinguished the 30 cell lines, we used t-SNE embedding to reduce the dimension of the convolutional features of CLCNet (e.g. 2048 dimensions) and visualized the processed features in $2 \mathrm{~d}$ space. The t-SNE plot of the 30 cell lines is shown in Fig. 4. Each dot represents one cell image and is colored by its cell line label. There were clear gaps between different cell lines which validates that CLCNet can find the decision boundary and distinguishes the 30 cell lines well. When we associated the cell lines' distribution with their incubation times, we found an interesting phenomenon that samples with similar times are mapped to 
adjacent areas. For example, in Fig. 5, the samples of HT1080 were clustered

well. Samples within 0-24 hours were close to the samples within 24-48 hours.

The complete results of the 30 cell lines with their incubation times are shown

in Fig. S3.

\section{Identify New Cell Lines}

The Confusion matrix of the transfer learning technique for identifying 14

new cell lines is shown in Fig. 6A. It is clear that integrating the transfer

learning with CLCNet can identify 14 new cell lines well with $96.5 \%$

accuracy. We showed the t-SNE plot of the 44 cell lines in Fig. 6B. The pink

dots show the data for the 30 cell line dataset and the additional colors show

the 14 new cell line samples. The samples of the 14 new cell lines were

mapped to the margin space between the 30 cell lines. By comparing Fig. 6A

with Fig. 6B, some misclassified cases can be explained. For example, in Fig.

$6 \mathrm{~A}, 98 \%$ of MCF7 images were classified accurately but $2 \%$ of data were

misclassified as ASPC1. In Fig. 6B, some dots of MCF7 (i.e. red box)

gathered with the ASPC1's dots which represents that the feature vectors of

MCF7 images are similar to that of ASPC1. In contrast, the well-classified

cases (i.e. green box) MRC5 and Min6 clustered well in the t-SNE plot and obtained the accuracy of $99 \%$. We showed the regression results for the test 
fold-1 of the 14 cell lines in Fig. 6C and the whole cross-validation results were presented in Fig. S4.

We demonstrated the advantages of the transfer learning method compared with the way of training model from scratch in Fig. 6D-E. The transfer learning method requires less computational resource and considerably less training time compared with training the whole model from scratch. The transfer learning method only spent 4 training epochs to loss convergence and its average training time is 0.078 hours. The overall performance of the transfer learning technique for identifying the 44 cell lines was shown in

Table. 2 .

\section{Discussion}

In summary, in the classification task of identifying 30 cell lines, our proposed computer-aided system CLCNet achieved the overall performance of $99.7 \%$ precision and the recall of $99.7 \%$. We compared the Xception backbone with three SOTA architectures, e.g. ResNet50, VGG19, MobileNet. The classification performance of the three deep networks has been validated in the previous studies on the analysis of cellular images [10-12]. In our experiments, the Xception model outperformed the other architectures, which can be attributed to the DSC blocks which decouple spatial correlations from cross-channel correlations and allows more efficient training. It should be noted that MobileNet also includes several DSC layers in its structure [22], however MobileNet is lightweight and targets the deployment on a mobile platform ahead of outright performance. Hence, we picked up the Xception as the backbone of CLCNet for classification. We used the t- 
SNE plots (Fig. 4) to visualize the high-dimensional features of CLCNet. It is clear that samples (dots) that belong to the same cell lines are clustered together and there are clear gaps between the 30 cell lines. These results proved that CLCNet is powerful for identifying the 30 cell lines.

The regression performance of CLRNet was satisfactory with an R2score $=0.931$. We also conducted an ablation experiment using the cell images to train Xception purely for regression. Here, the Xception architecture is almost the same as Fig. S1 except for the final FC layer. The FC only includes one neuron and the used loss function is MSE. The regression results of the ablation model are shown in Fig. S5 and the performance is really poor. Because in our dataset, some cell images from different cell lines have the same time labels which affects the training of the network. For example, we collected two cell images from A431 and A549 cell lines separately but these two images have the same incubation times (e.g. Fig. 1). There are many differences between the two cell images, e.g. the single cell morphology, cell counts, and confluency. The two images have completely different appearances but take the same time labels, which influences the feature learning of the network. From Fig. 5, we observed that the convolutional features of CLCNet include information about the incubation times. Hence, we took the features of CLCNet as the input of CLRNet to predict the incubation times for cell images. The above results validated our strategy is effective for the regression task.

Aiming to handle data from new cell lines, we integrated the transfer learning technique with our proposed framework. We established a smallscale dataset that included 14 cell lines to evaluate the method's performance. From the pre-trained model side, the 14 new cell lines were unseen so the model cannot output the matching labels for these images. Considering the pre-trained CLCNet has learned good representations from the data of 30 cell lines, we fixed the parameters of all layers except for the final FC 
layer. The FC were re-trained by using the combined dataset (i.e. training set from 30 cell lines + training set from 14 cell lines). The classification accuracy for the 14 cell lines reached $96.5 \%$ and the performance of identifying the 30 cell lines was not influenced ( $99.8 \%$ accuracy) by re-training. Although the transfer learning technique needs to re-train the part of CLCNet, its training speed was 480 times faster than training the model from scratch. These results suggested that the transfer learning method is possible to solve the issue.

In this paper we have established a proof of concept for how an image-based AI method can be used for cell line authentication, including the use of transfer learning to extend classification to cell lines for which we have only a small number of example images. In doing so we have identified a number of areas for future research. Firstly the ability to identify and quantify contamination of cell lines with small amounts of another cell line would be a useful functionality. Secondly, having to retrain the model when adding new cell lines might be a drawback as greater numbers of cell lines are built up. Some new techniques like conformal prediction [를 or open set recognition [24] can automatically decide data is seen or unseen without extra model training. These methods can potentially be integrated with the transfer learning method to solve this issue. There is also the question of how much training data is necessary: How many cell lines are required for the base training (is 30 cell lines enough)? How many cell lines can this be expanded to via transfer learning (200 cell lines or more) whilst maintaining a satisfactory level of performance? Finally, we have demonstrated a proof of concept for rapid cell line authentication. When this system is deployed into the laboratory, the development of guidelines for interpreting the confidence value outputted by CLCNet, and the incubation time predicted by CLRNet, how to assess performance and how to integrate with current laboratory practice are required. 
In this study, we have attempted to automatically authenticate cell lines by using deep neural networks on brightfield images. We proposed a novel multi-task cell image recognition framework to authenticate cell lines and predict the duration of incubation. We established a large dataset consisting of 47671 brightfield images of 30 cell lines. The classification and regression performance on the dataset were excellent. We also integrated transfer learning with our proposed system to identify new cell lines and obtained $96.7 \%$ accuracy. These results demonstrated that our proposed framework can effectively authenticate the identities of cell lines on brightfield images.

\section{Data availability}

The datasets generated and/or analyzed during the current study are not publicly available due AstraZeneca Licenses but are available from the corresponding author on reasonable request. Source code from this project and our model can be accessed from GitHub: https://github.com/BIPL-UoL/AnAutomated-Cell-Line-Authentication-Method-for-AstraZeneca-Global-Cell$\underline{\text { Bank }}$

\section{Acknowledgments}

The authors would like to thank the AZ UKCCB team for the provision of the cell line samples and images.

\section{Funding}

Authors L.T. is sponsored by University of Leicester GTA studentship (GTA 2020), China Scholarship Council and AstraZeneca - University Leicester collaboration agreement (CR-019972). Authors (A.C., N.K., K.H., J.O., Y.W.) are employees of AstraZeneca. AstraZeneca provided the funding for this research and provided support in the form of salaries for all authors, but did not have any additional role in the study design, data collection and analysis, decision to publish, or preparation of the manuscript. The specific roles of these authors are articulated in the 'author contributions' section. 


\section{Author Contributions}

L.T., A.C., N.R.K., K.H., Y.W. and H.Z. designed and performed research; L.T. and A.C. performed development of methodology; L.T., A.C., N.R.K. K.H. and Y.W. wrote the paper; N.R.K. and K.H. provided acquisition, analysis and interpretation of data, and statistical analysis; L.T., A.C., N.R.K., K.H., J.O., Y.W. and H.Z. performed review and revision of the paper. All authors read and approved the final paper.

\section{Compliance with ethical standards}

\section{Conflict of Interest}

The authors declare that they have no conflict of interest.

\section{Ethical Approval and Consent to Participate}

The authors declare this study don does not require ethical approval.

\section{Publisher's note}

Springer Nature remains neutral with regard to jurisdictional claims in published maps and institutional affiliations. 
1. Masters JR, Thomson JA, Daly-Burns B, Reid YA, Dirks WG, Packer $P$ et al. Short tandem repeat profiling provides an international reference standard for human cell lines. Proc Natl Acad Sci U S A 98, 8012-8017 (2001).

2. Ioannidis JP. Why most published research findings are false. PLoS Med 2, e124 (2005).

3. Boonstra JJ, Van Marion R, Beer DG, Lin L, Chaves P, Ribeiro C et al. Verification and unmasking of widely used human esophageal adenocarcinoma cell lines. J Natl Cancer Inst 102, 271-274 (2010).

4. Burden N, Sewell F, and Chapman K. Testing chemical safety: what is needed to ensure the widespread application of non-animal approaches? PLoS Biol 13, e1002156 (2015).

5. Almeida JL, Cole KD, and Plant AL. Standards for cell line authentication and beyond. PLoS Biol 14, e1002476 (2016).

6. Reid Y, Storts D, Riss T, and Minor L. Authentication of human cell lines by STR DNA profiling analysis. Bethesda(MD): NCBI, 2013.

7. Parson W, Kirchebner R, Mühlmann R, Renner K, Kofler A, Schmidt $S$ et al. Cancer cell line identification by short tandem repeat profiling: power and limitations. FASEB J 19, 1-18 (2005).

8. Chatterjee R. Cell biology. Cases of mistaken identity. Science 315, 928-931 (2007).

9. Sharma SV, Haber DA, and Settleman J. Cell line-based platforms to evaluate the therapeutic efficacy of candidate anticancer agents. Nat Rev Cancer 10, 241-253 (2010).

10. Mzurikwao D, Khan MU, Samuel OW, Cinatl J, Wass M, Michaelis $\mathrm{M}$ et al. Towards image-based cancer cell lines authentication using deep neural networks. Sci Rep 10, 1-15 (2020).

11. Oei RW, Hou G, Liu F, Zhong J, Zhang J, An Z et al. Convolutional neural network for cell classification using microscope images of intracellular actin networks. PLoS One 14, e0213626 (2019).

12. Akogo DA, Appiah V, and Palmer X-L. CellLineNet: end-to-end learning and transfer learning for multiclass epithelial breast cell line classification via a convolutional neural network. Preprint at https://arxivorg/abs/180806041, (2018).

13. Press WH, Teukolsky SA, Flannery BP, and Vetterling WT. Numerical recipes in Fortran 77: volume 1, volume 1 of Fortran numerical recipes: the art of scientific computing. Cambridge: Cambridge university press, 1992.

14. Chollet F. Xception: Deep learning with depthwise separable convolutions. In: Proceedings of the IEEE conference on computer vision and 
https://ieeexplore.ieee.org/document/8099678.

15. Ioffe S and Szegedy C. Batch normalization: Accelerating deep network training by reducing internal covariate shift. In: International conference on machine learning. 2015. https://dl.acm.org/doi/abs/10.5555/3045118.3045167.

16. Kaiser L, Gomez AN, and Chollet F. Depthwise separable convolutions for neural machine translation. Preprint at https://arxivorg/abs/170603059, (2017).

17. Hastie T, Tibshirani R, and Friedman J. The elements of statistical learning: Data mining, inference and prediction. New York: Springer-Verlag, 2001. p. 552.

18. Srivastava N, Hinton G, Krizhevsky A, Sutskever I, and Salakhutdinov R. Dropout: a simple way to prevent neural networks from overfitting. J Mach Learn Res 15, 1929-1958 (2014).

19. Tong L, Zhang Q, Sadka A, Li L, and Zhou H. Inverse boosting pruning trees for depression detection on Twitter. Preprint at https://arxivorg/abs/190600398, (2019).

20. Paszke A, Gross S, Massa F, Lerer A, Bradbury J, Chanan G et al. Pytorch: An imperative style, high-performance deep learning library. Adv Neural Inf Process Syst 32, 8026-8037 (2019).

21. Pedregosa F, Varoquaux G, Gramfort A, Michel V, Thirion B, Grisel $\mathrm{O}$ et al. Scikit-learn: Machine learning in Python. J Mach Learn Res 12, 2825-2830 (2011).

22. Sandler M, Howard A, Zhu M, Zhmoginov A, and Chen L-C. Mobilenetv2: Inverted residuals and linear bottlenecks. In: Proceedings of the IEEE conference on computer vision and pattern recognition. 2018. https://ieeexplore.ieee.org/document/8578572.

23. Shafer G and Vovk V. A Tutorial on Conformal Prediction. J Mach Learn Res 9, (2008).

24. Geng C, Huang S-j, and Chen S. Recent advances in open set recognition: A survey. IEEE Trans Pattern Anal Mach Intell, (2020). 


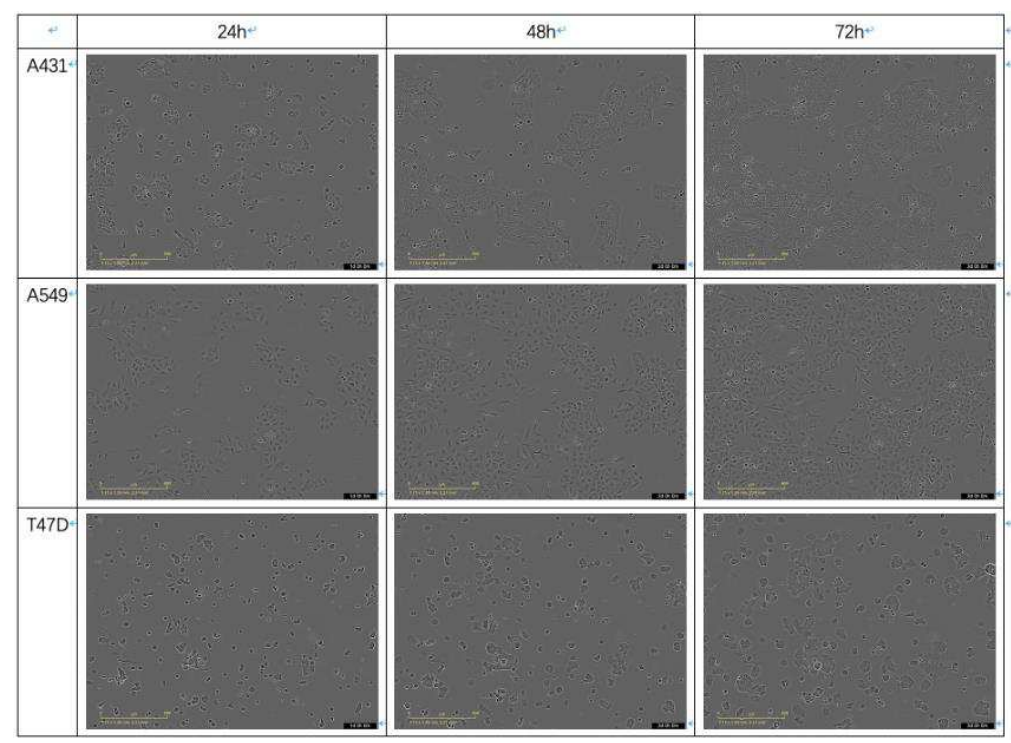

Fig. 1 Example images of three cell lines with different incubated durations (hours). In the three examples shown here, 3 discrete time points were taken for A549, A549, T47D at 24, 48 and 72 hours. With the increased amount of the incubation time, we observed increased cell counts and confluency and a formation of colonies. Notable single cell morphology can also be observed, e.g. A549 cells are more elongated in shape compared to A431 cells, whereas T47D cells are typically larger in size and round. 


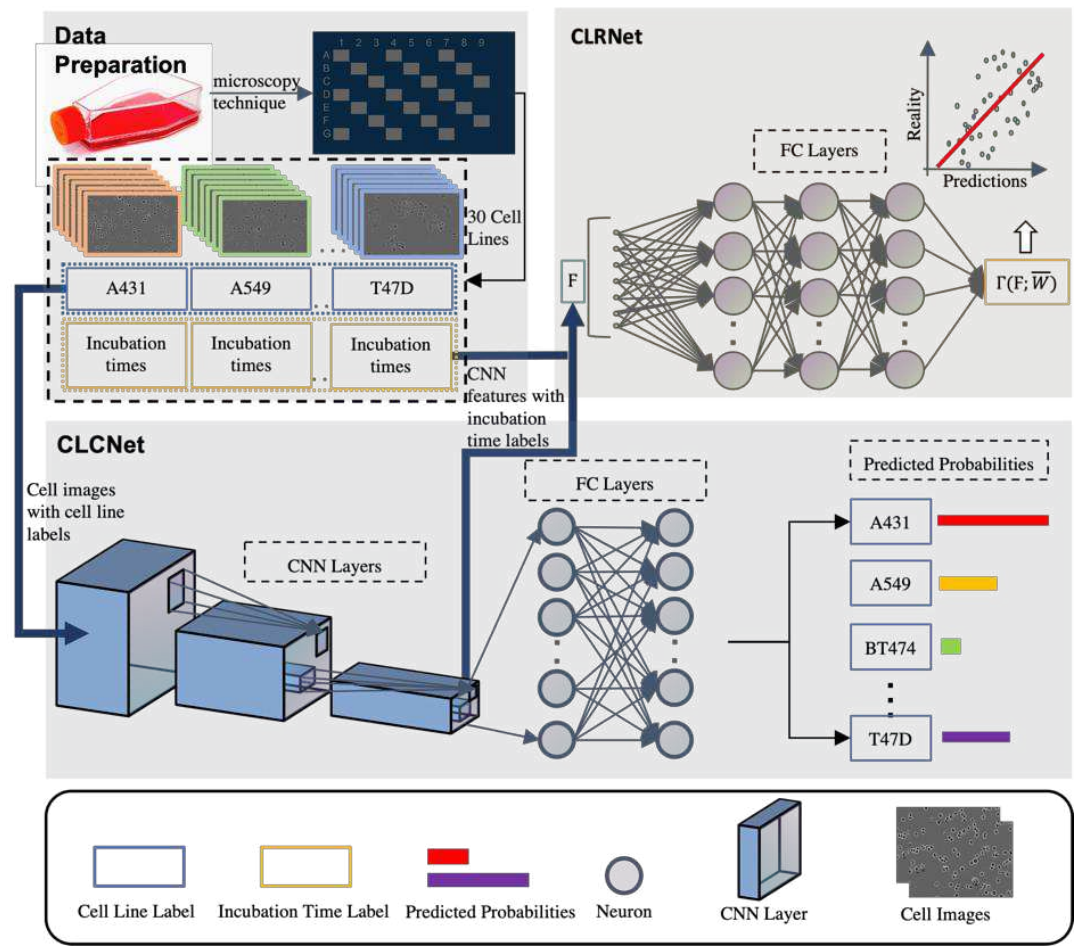

Fig. 2 The framework of the proposed automated cell line authentication system. In the data preparation stage, cell images are collected using the high-throughput IncuCyte microscopy technique from 30 cell lines and each cell image has two separate labels, e.g. cell line name and incubation time. Then, the deep learning network CLCNet learns the image-level features from the input cell images with their cell line labels and outputs predicted classes for test cell images. Once CLCNet is trained, the convolutional features of the training data are extracted to train CLRNet. CLRNet predicts the times of how long cell lines have been incubated simultaneously. 


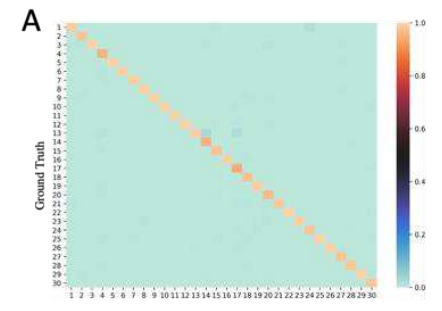

ResNet50

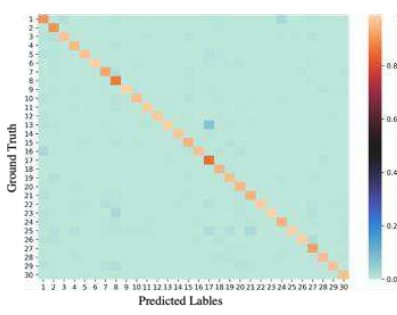

MobileNet

B

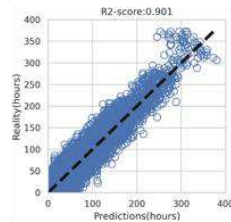

ResNet50

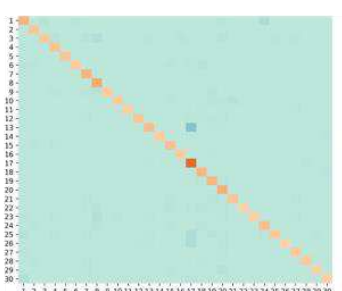

VGG19

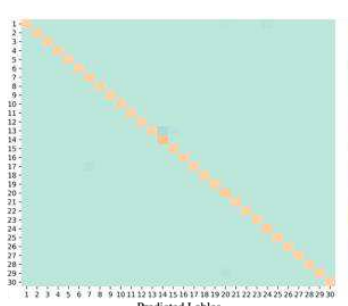

Xception

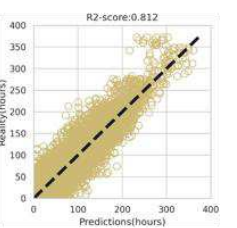

MobileNet

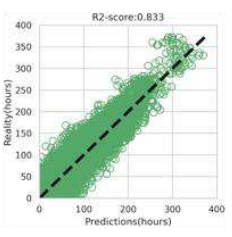

VGG19 $\begin{array}{lll}1 . & \text { A43 } \\ 2 . & \text { A549 } \\ 1 & 3 . & \text { BT4 }\end{array}$ BT474 HCC 2279
HCC1395 HCC70 HCC78 HCC 827 HS-5 HT 1080 Hop62 KELLY KPL4 UU99 LUHMES MCF10A MDA MB 23 Mia Paca2 NCI H1933 $\mathrm{NCI} \mathrm{H} 1975$ NCI H2030 NCI H2073 PC3 SKBR3 SNU601

Fig. 3 Visualization of the classification and regression results on the 30 cell lines' dataset. A. Confusion matrices of the 30-category classification. The corresponding cell line names of the coordinates are shown in the right legend. Compared with ResNet50, VGG19 and MobileNet, Xception model made fewer prediction errors B. Scatter plots of the predicted incubated durations vs. the real incubated durations. Here we show the prediction results on the test fold- 1 as an example; the complete results of cross-validation are shown in Fig. S2. Xception outperformed other three methods in predicting the amount of incubation time with R2-score $=0.939$. 


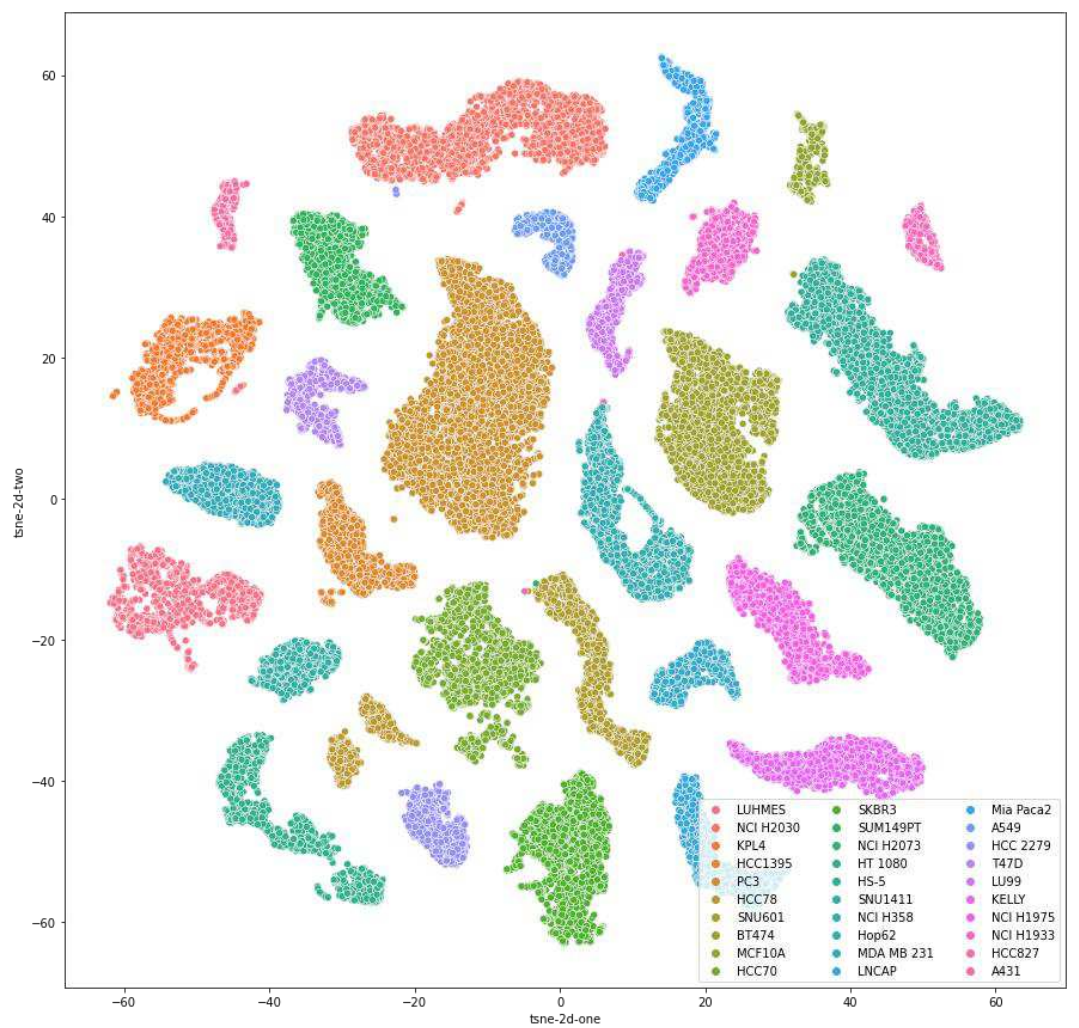

Fig. 4 t-SNE Embedding of our 30 cell lines. The t-SNE tool reduces the dimension of the CLCNet's convolutional features and visualizes the processed features in 2D space. Each dot represents one cell image and is colored by its matching cell line name. There were clear gaps between different cell lines, which validates that CLCNet model can distinguish the 30 cell lines well.

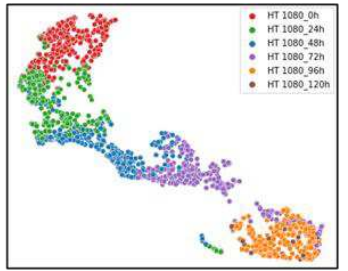

HT1080

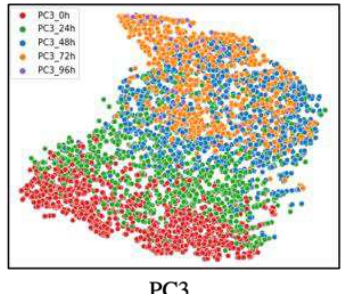

PC3

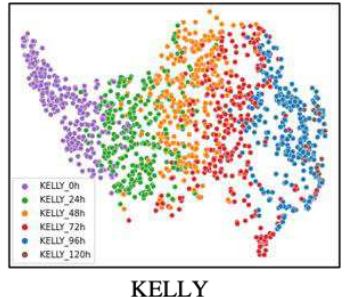

KELLY

Fig. 5 t-SNE plots of 3 example cell lines (e.g. HT1080, PC3, KELLY). These plots enlarge the three cell lines' distribution of Fig.4. Each dot is colored by the range of the incubation duration (e.g. 0-24 hours, 24-48 hours). An interesting phenomenon was found that samples with similar times locate in adjacent areas. For example, the samples of HT1080 were clustered well and samples within 0-24 hours were close to the samples within 24-48 hours. 


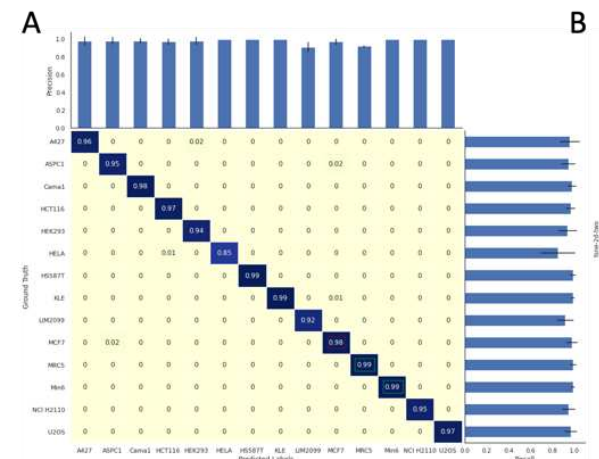

C

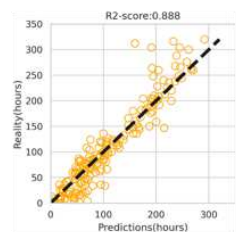

D

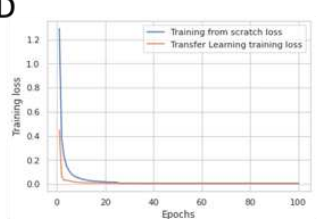

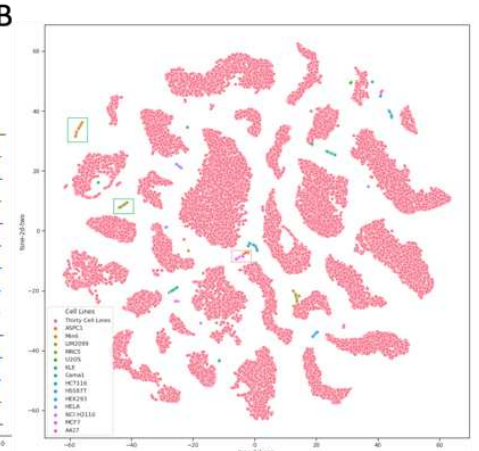

$E$

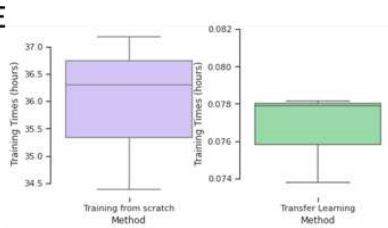

Fig. 6 Performance of the transfer learning technique for identifying 14 new cell lines.

A. Confusion matrix of the 14 cell line classification B. t-SNE plot of 44 cell lines. The pink dots are the data of 30 cell lines and dots of other colors are the data of 14 new cell lines. It can be seen that the samples of the 14 new cell lines were mapped to the margin space between the 30 cell lines $\mathbf{C}$. Regression results for the test fold-1 of the cross-validation D. Comparison of the convergence speed between full model training from scratch and final layer fine-tuning using the transfer learning. "Training from scratch" means that all layers of Xception are re-trained using the dataset $\mathbf{E}$. Comparison of the training times 
Tables

\begin{tabular}{|c|cccc|cc|}
\hline & \multicolumn{5}{|c|}{ Classification } & \multicolumn{2}{c|}{ Regression } \\
\hline Backbones & Accuracy & Precision & Recall & F1-score & $M S E$ & $R 2$-score \\
\hline ResNet50 & $0.987 \pm 0.002$ & $0.984 \pm 0.002$ & $0.987 \pm 0.003$ & $0.985 \pm 0.002$ & $452.533 \pm 54.547$ & $0.880 \pm 0.015$ \\
\hline VGG19 & $0.975 \pm 0.004$ & $0.968 \pm 0.004$ & $0.972 \pm 0.003$ & $0.972 \pm 0.003$ & $677.637 \pm 43.709$ & $0.821 \pm .0013$ \\
\hline MobileNet & $0.965 \pm 0.001$ & $0.952 \pm 0.002$ & $0.960 \pm 0.002$ & $0.956 \pm 0.002$ & $769.111 \pm 44.984$ & $0.797 \pm 0.013$ \\
\hline Xception & $\mathbf{0 . 9 9 8} \pm \mathbf{0 . 0 0 1}$ & $\mathbf{0 . 9 9 7} \pm \mathbf{0 . 0 0 1}$ & $\mathbf{0 . 9 9 7} \pm \mathbf{0 . 0 0 1}$ & $\mathbf{0 . 9 9 7} \pm \mathbf{0 . 0 0 1}$ & $\mathbf{2 6 2 . 2 8 3} \pm \mathbf{3 2 . 0 8 5}$ & $\mathbf{0 . 9 3 1} \pm \mathbf{0 . 0 0 8}$ \\
\hline
\end{tabular}

Table. 1 Classification and Regression Results on the 30 cell lines dataset: [Mean

Value \pm Standard Deviation] by four deep networks. R2-score: coefficient of determination regression score. The best results are shown in bold. 


\begin{tabular}{|c|c|c|c|}
\hline Metrics & 14 Cell Lines & 30 Cell Lines & Overall \\
\hline Accuracy & $0.965 \pm 0.018$ & $0.998 \pm 0.001$ & $0.997 \pm 0.001$ \\
\hline Precision & $0.977 \pm 0.012$ & $0.997 \pm 0.001$ & $0.991 \pm 0.004$ \\
\hline Recall & $0.958 \pm 0.024$ & $0.996 \pm 0.001$ & $0.984 \pm 0.008$ \\
\hline F1-score & $0.968 \pm 0.017$ & $0.997 \pm 0.001$ & $0.987 \pm 0.006$ \\
\hline MSE & $526.230 \pm 62.090$ & $232.690 \pm 0.000$ & $263.360 \pm 3.287$ \\
\hline R2-score & $0.853 \pm 0.017$ & $0.939 \pm 0.000$ & $0.932 \pm 0.001$ \\
\hline
\end{tabular}

Table. 2 Classification and Regression Results of the transfer learning technique for identifying the 44 cell lines. The columns of the "14 cell lines" represented the data of 14 cell lines is split from the test dataset during evaluation. The columns of the " 30 cell lines" represented the data of 30 cell lines is split from the test dataset during evaluation. The "overall" column showed the results for the whole test set (44 cell lines). 


\section{Supplementary Figures}

Fig. S1 The Structure of the Xception Model. Conv is the convolution layer, BatchNorm is the batch normalization, ReLU is the activation function, SeparableConv is the depthwise separable convolution layer, MaxPool is the Maxpooling layer, and FC is the fully connected layer.

Fig. S2 Regression Results of four backbones(e.g. ResNet50, VGG19, MobileNet, Xception) on 5-fold Cross-Validation. Scatter plots of the predicted incubated durations vs. the real incubated durations.

Fig. S3 TSNE plots of 30 cell lines. Each dot is colored by the range of the incubation duration (e.g. 0-24 hours, 24-48 hours).

Fig. S4 Regression Results of transfer learning method for 14 new cell lines on 5-fold Cross-Validation. Scatter plots of the predicted incubated durations vs. the ground truth incubated durations.

Fig. S5 Failure cases of training Xception purely with raw cell images for regression.

\section{Supplementary Tables}

Table. S1 List of 30 cell lines. All base medium was purchased from Sigma Aldrich, and supplemented with 10\% FBS (Sigma Aldrich) and 1x GlutaMAX (Gibco) unless otherwise stated.

Table. S2 List of 14 cell lines. 


\section{Supplementary Files}

This is a list of supplementary files associated with this preprint. Click to download.

- Supplementary.pdf 